\title{
ON SPENCER'S COHOMOLOGY THEORY FOR LINEAR PARTIAL DIFFERENTIAL OPERATORS
}

\author{
BY \\ JOSEPH JOHNSON
}

\begin{abstract}
Let $D$ be a linear partial differential operator between vector bundles on a differentiable manifold $X$ of dimension $n$. Let $\mathfrak{D}$ be the sheaf of germs of differentiable functions on $X$. For every $h \in Z$ a spectral sequence $\left({ }^{h} E^{p q}\right)$ is associated to $D$. When $D$ satisfies appropriate regularity conditions these spectral sequences degenerate for all sufficiently large $h$ and ${ }^{h} E_{2}^{p 0}$ is the $p$ th Spencer cohomology for $D$. One can compute ${ }^{h} E_{2}^{p q}$ as the cohomology at $\Lambda^{p} T^{*} \otimes \mathcal{D} R_{h-p, q}$ of a complex

$$
0 \rightarrow R_{h q} \rightarrow \Lambda^{1} T^{*} \otimes_{\mathcal{O}} R_{h-1, q} \rightarrow \cdots \rightarrow \Lambda^{n} T^{*} \otimes_{\mathcal{O}} R_{h-n, q} \rightarrow 0 .
$$

When $q=0$ this complex coincides with the usual (first) Spencer complex for $D$.

These results give a generalization of Spencer's theory. The principal importance of this generalization is that it greatly clarifies the role played by homological algebra in the theory of overdetermined systems of linear partial differential equations.
\end{abstract}

1. Introduction. This paper was written to show that the theory of linear differential operators introduced by Spencer (see [2]) is in part a special case of a more general theory of homological algebra.

Let us fix our notation: $X$ is a differentiable $\left({ }^{1}\right)$ manifold of dimension $n ; \mathfrak{D}$ is the sheaf of germs of differentiable functions on $X\left({ }^{2}\right)$; $\mathfrak{D}$ is the sheaf of germs of linear differential operators of $\mathfrak{D}$ into itself. Let $\mathfrak{\subseteq}$ be the category of left $\mathfrak{D}$-modules. Denote by $T$ the sheaf of germs of differentiable vector fields on $X$.

Let $E$ and $F$ be fixed vector bundles( $\left.{ }^{3}\right)$ on $X$ and $D: E \rightarrow F$ a linear differential operator. Consider the problem of solving the equation

$$
D(?)=f
$$

Received by the editors January 21, 1970.

AMS 1970 subject classifications. Primary 35N10, 47F05, 58G99; Secondary $18 \mathrm{G} 40$.

Key words and phrases. Linear partial differential operator, formally integrable operator, over-determined system of linear partial differential equations, Spencer cohomology.

${ }^{(1)}$ Differentiable will always mean of class $C^{\infty}$. Maps, vector bundles over differentiable manifolds, etc. will always be assumed to be differentiable. In addition it will be assumed that $X$ is paracompact.

$\left({ }^{2}\right)$ All tensor products will be over $\mathfrak{D}$. Also $\mathfrak{D}$ has two distinct $\mathfrak{D}$-module structures accordingly as one places the sections of $\mathfrak{D}$ on the left of the sections of $\mathscr{D}$ or on the right in performing the multiplication. The position of $\mathscr{D}$ in any tensor product will always indicate which $\mathfrak{O}$-module structure is being used.

$\left(^{3}\right)$ If a vector bundle is represented by a certain letter, that same letter will often be used to denote the associated sheaf of germs of differentiable sections as well.

Copyright $(1)$ 1971, American Mathematical Society 
when $f$ is a given global section of $F$. It was shown in [1] that there is a morphism of left $\mathfrak{D}$-modules

$$
D^{*}: \mathfrak{D} \otimes F^{*} \rightarrow \mathfrak{D} \otimes E^{*}
$$

obtained from $D$. Call $D^{*}$ the adjoint morphism and its cokernel $M($ resp. kernel $N$ ) the adjoint cokernel (resp. adjoint kernel). The induced map

$$
\operatorname{Hom}_{\mathfrak{D}}\left(\mathfrak{D} \otimes E^{*}, \mathfrak{D}\right) \rightarrow \operatorname{Hom}_{\mathfrak{D}}\left(\mathfrak{D} \otimes F^{*}, \mathfrak{D}\right)
$$

can be identified with the map

$$
\Gamma(X, E) \rightarrow \Gamma(X, F)
$$

given by $D$. An obvious necessary condition for $D(?)=f$ to have a solution is that $f$, considered as an element of $\operatorname{Hom}_{D}\left(\mathfrak{D} \otimes F^{*}, \mathfrak{D}\right)$, should vanish on $N$.

Applied to

$$
0 \rightarrow \mathfrak{D} \otimes F^{*} / N \rightarrow \mathfrak{D} \otimes E^{*} \rightarrow M \rightarrow 0
$$

the long exact sequence for ExtD in the first variable gives a diagram

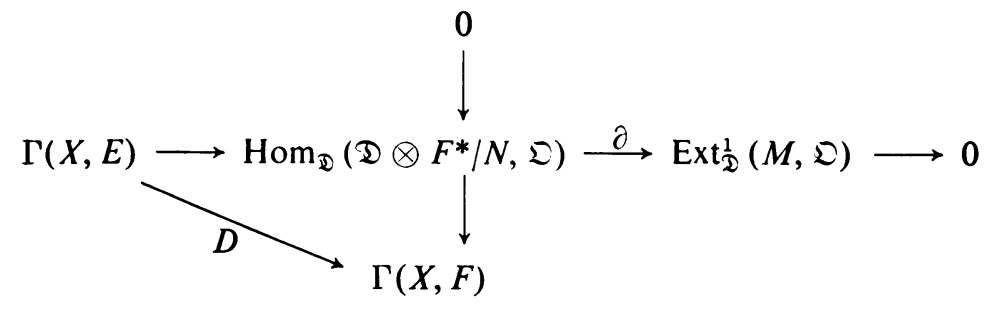

with rows and columns exact. Thus given a section $f$ of $F$ satisfying an "integrability condition", i.e. lying in $\operatorname{Hom}_{\mathfrak{D}}\left(\mathfrak{D} \otimes F^{*} / N, \mathfrak{D}\right)$ (vanishing on $\left.N\right)$, there is an element $\partial(F)$ in $\operatorname{Ext}_{\mathfrak{D}}^{1}(M, \mathfrak{D})$ which is zero if and only if $D(?)=f$ has a solution. (See the Proposition in $\S 1$ of [1].) Thus $\operatorname{Ext}_{\mathfrak{D}}^{1}(M, \mathfrak{D})$ is the space of all "obstructions" to solving equations $D(?)=f$ when $f$ ranges over the sections of $F$ which satisfy the integrability condition.

The importance of $\operatorname{Ext}_{\mathfrak{D}}^{1}(M, \mathfrak{D})$ thus established, the next obvious task is to seek theorems that give us information about it. In [1] it was shown that there is a spectral sequence $\left(E_{r}^{p q}\right)_{r=2,3, \ldots}$ converging to $\operatorname{Ext}_{\mathfrak{D}}^{p+q}(M, \mathfrak{D})$.

It can be obtained by representing the functor $M \mapsto \operatorname{Hom}_{\mathcal{D}}(M, \mathfrak{D})$ as a composite of two functors. For the first of these we can take $M \mapsto \operatorname{Hom}_{\mathfrak{O}}(M, \mathfrak{D})$ of $\mathbb{C}$ into itself. For the second we can take the covariant functor from $\mathbb{E}$ into the category of abelian groups which takes $P$ into the global constants $C(P)$ of $\Gamma(X, P)$. (This method is slightly different from that used in [1].) We then obtain from this a spectral sequence converging to $\operatorname{Ext}_{\mathfrak{D}}^{p+q}(M, \mathfrak{D})$ with

$$
E_{2}^{p q}=R^{p} C\left(\operatorname{Ext}_{\mathfrak{D}}^{q}(M, \mathfrak{D})\right)
$$


where $R^{p} C$ is the $p$ th right derived functor of $C$. It will be shown shortly that the vector spaces $R^{p} C\left(\operatorname{Hom}_{\mathfrak{O}}(M, \mathfrak{D})\right)$ are a fundamental object of study of the "infinite" Spencer theory.

In [2], Spencer has defined complexes

$$
S_{h}: 0 \rightarrow R_{h} \rightarrow \Lambda^{1} T^{*} \otimes R_{h-1} \rightarrow \cdots \rightarrow \Lambda^{n} T^{*} \otimes R_{h-n} \rightarrow 0 .
$$

The $R_{h}$ are related to the adjoint morphism $D^{*}$ as follows. Let $k$ be the order of $D$. We have a commutative diagram

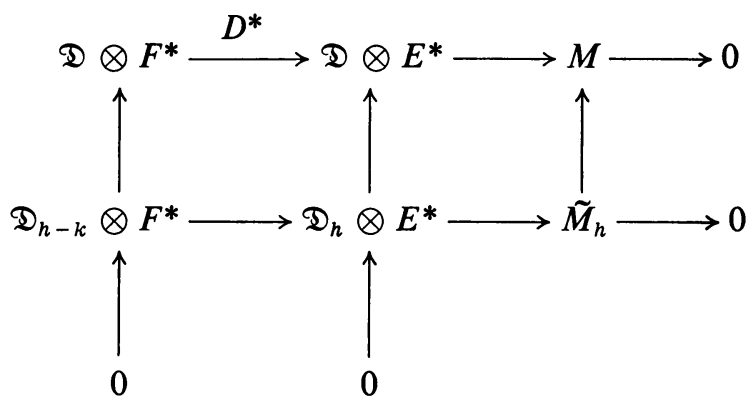

with exact rows and columns. Here $\mathfrak{D}_{h}$ represents the sheaf of germs of sections of $\mathfrak{D}$ of order $\leqq h$ and $\tilde{M}_{h}$ is the cokernel of the obvious morphism. Then $R_{h}=$ $\operatorname{Hom}_{\mathfrak{O}}\left(\tilde{M}_{h}, \mathfrak{D}\right)$ and $R_{\infty}=\operatorname{Hom}_{\mathfrak{D}}(M, \mathfrak{D})$. Let $\mathfrak{D}_{\infty}=\mathfrak{D}$ and $\tilde{M}_{\infty}=M$. It was shown in [1] that the $p$ th cohomology of the complex $\Gamma\left(X, S_{\infty}\right)$ is $R^{p} C\left(\operatorname{Hom}_{\mathcal{D}}(M, \mathfrak{D})\right)$ (Theorem 2).

Let us note that when $D$ is formally integrable the theory of [1] tells us that $H^{p}\left(\Gamma\left(X, S_{\infty}\right)\right)$ is $\operatorname{Ext}_{D}^{p}(M, \mathfrak{D})$. In fact in this case $M$ is locally free and $E_{2}^{p q}=0$ when $q \neq 0$. It follows from the spectral sequence that $R^{p} C\left(\operatorname{Hom}_{\mathcal{D}}(M, \mathfrak{D})\right)$ is $\operatorname{Ext}_{D}^{p}(M, \mathfrak{D})$. From a preceding remark,

$$
H^{p}\left(\Gamma\left(X, S_{\infty}\right)\right)=R^{p} C\left(\operatorname{Hom}_{\mathfrak{D}}(M, \mathfrak{D})\right)=\operatorname{Ext}_{\mathfrak{D}}^{p}(M, \mathfrak{D}) .
$$

Thus for a formally integrable operator, the complex $S_{\infty}$ contains all the desired information. There is however a major problem in using $S_{\infty}$. Its terms are sheaves of vector spaces and that is all. In general they are not finite dimensional. The theory of [1] is plagued with the generalization of that problem; namely $\operatorname{Ext}_{\mathfrak{D}}^{p}(M, \mathfrak{D})$ is only a sheaf of infinite-dimensional vector spaces.

What is given here is a refinement of the theory of [1] which produces results of the type indicated above but for the finite Spencer complexes $S_{h}, h<\infty$, as well as for $S_{\infty}$. The ideas of [1] are adequate to do this. However they need to be applied to a category other than $\mathfrak{E}$. The category $\mathfrak{A}$ described in the next section has the needed advantage that $\tilde{M}=\bigoplus_{h \in Z} \tilde{M}_{h}$ can be considered as an object of it.

The consequences of applying the methods of [1] to $\mathfrak{A}$ will now be summarized. Let

$$
R_{h q}=\mathbf{E x t}_{\mathfrak{D}}^{q}\left(\tilde{M}_{h}, \mathfrak{D}\right)
$$


There exist complexes (generalized Spencer complexes)

$$
S_{h q}: 0 \rightarrow R_{h q} \rightarrow \Lambda^{1} T^{*} \otimes R_{h-1, q} \rightarrow \cdots \rightarrow \Lambda^{n} T^{*} \otimes R_{h-n, q} \rightarrow 0
$$

with $S_{h 0}=S_{h}$. There are also spectral sequences $\left({ }^{h} E_{r}^{p q}\right)_{r=2,3, \ldots}$ with initial terms

$$
{ }^{n} E_{2}^{p q}=H^{p}\left(\Gamma\left(X, \Lambda \cdot T^{*} \otimes R_{h-\cdot, q}\right)\right) .
$$

If $M$ has the proper kind of resolution in $\Re$, the spectral sequence $\left({ }^{n} E_{r}^{p q}\right)$ converges to $\operatorname{Ext}_{\mathfrak{D}}^{p+q}(M, \mathfrak{D})$ for all sufficiently large $h$ in $Z$.

If $D$ is formally integrable, $M$ has the required type of resolution. As a consequence the following is true for all sufficiently large $h$ in $Z$ :

(1) $R_{h-p, q}=0$ if $0 \leqq p \leqq n$ and $q \neq 0$.

(2) $\operatorname{Ext}_{\mathfrak{D}}^{p}(M, \mathfrak{D})$ is the $p$ th cohomology of the complex $\Gamma\left(X, S_{h}\right)$.

2. The category $\mathfrak{A}$. Let $\mathfrak{D}_{r}$ be the sheaf of germs of sections of $\mathfrak{D}$ which are of order $\leqq r$. Let $\mathfrak{D}$ be the direct sum $\mathfrak{D}=\bigoplus_{r \in Z} \mathfrak{D}_{r}$. Then $\mathfrak{D}$ is in a natural way a sheaf of graded rings because $\mathfrak{D}_{r} \mathfrak{D}_{s} \subset \mathfrak{D}_{r+s}$.

The category $\mathfrak{R}$ is to have graded left $\tilde{D}$-modules for its objects. A morphism of $\mathfrak{R}$ will be any morphism of left $\tilde{\mathfrak{D}}$-modules which is homogeneous of degree zero.

There are various interesting ways of defining objects of $\AA$. One is to consider $\mathfrak{D}$ as a filtered ring (using order) and to consider any filtered left $\mathfrak{D}$-module $L$. Then $\oplus_{\mathrm{r} \in \boldsymbol{Z}} L_{r}$ is in a natural way a graded left $\mathfrak{D}$-module. A more important example from our point of view can be obtained from the linear differential operator $D$.

Namely let $k$ be the order of $D$ and consider the adjoint morphism $D^{*}$ : $\mathfrak{D} \otimes F^{*} \rightarrow \mathfrak{D} \otimes E^{*}$. By restriction we can obtain induced $\mathfrak{D}$-module morphisms

$$
D_{l}^{*}: \mathfrak{D}_{l} \otimes F^{*} \rightarrow \mathfrak{D}_{k+l} \otimes E^{*}
$$

for every $l \geqq 0$. Let $\tilde{M}_{k+l}$ be the cokernel of $D_{l}^{*}$ and let $\tilde{M}_{r}=\mathfrak{D}_{r} \otimes E^{*}$ if $r<k$. It is easy to see that the $\mathfrak{D}$-module morphisms $\mathfrak{D}_{s} \otimes \mathfrak{D}_{r} \otimes E^{*} \rightarrow \mathfrak{D}_{r+s} \otimes E^{*}$ induce morphisms $D_{s} \otimes \tilde{M}_{r} \rightarrow \tilde{M}_{r+s}$ From this it follows quickly that $\oplus_{r \in Z} \tilde{M}_{r}$ is an object of $\Re$.

Observe that the dual of the exact sequence of $\mathfrak{D}$-modules

$$
\mathfrak{D}_{l} \otimes F^{*} \rightarrow \mathfrak{D}_{k+l} \otimes E^{*} \rightarrow \tilde{M}_{k+l} \rightarrow 0
$$

is the exact sequence

$$
0 \rightarrow R_{k+l} \rightarrow J_{k+l}(E) \rightarrow J_{l}(F)
$$

of [2, p. 185]. This fact will be useful in connecting the theory developed here with the theory of [2].

The global section 1 of $\mathfrak{D}$, since it is also a section of $\mathscr{D}_{r}$ for every $r \geqq 0$, can be considered as a section of $\tilde{D}$ in various ways. To avoid confusion on this point, let $\gamma^{r}$ for $r \geqq 0$ be the section 1 of $\mathfrak{D}_{r}$ considered as a section of $\mathfrak{D}$ homogeneous of 
degree $r$. Observe that $\gamma$ is in the center of $\tilde{\mathfrak{D}}$. In general when we wish to consider a section $f$ of $D_{s}$ as a section of $(\mathfrak{D})_{r}$ with $r \geqq s$, let us write it as $\gamma^{r} f$ to indicate this. In particular the section $\delta$ of $T$ when considered as a section of $(\tilde{D})_{1}$ will be written $\gamma \delta$.

If $P$ is any object of $\Re$ and if $s \geqq r, \gamma^{s-r}$ maps $P_{r}$ into $P_{s}$ so that we have a direct system of $\mathfrak{D}$-modules $\left(P_{r}\right)_{r \in Z}$. Let $P_{\infty}=\operatorname{inj} \lim _{r} P_{r}$. It is easy to check that $P_{\infty}$ has a unique structure of left $\mathfrak{D}$-module such that for all $r, s \in Z$,

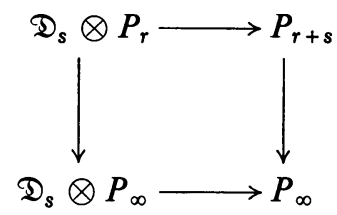

commutes.

If $r$ is in $Z$, define an object $\tilde{D}(r)$ of $\mathfrak{R}$ which as a left $\tilde{\mathfrak{D}}$-module is isomorphic to $\mathfrak{D}$, but which is graded by the rule $(\tilde{D}(r))_{s}=(\mathfrak{D})_{r+s}$. It is clear that $(\tilde{D}(r))_{\infty}=\mathfrak{D}$ and that the $\mathfrak{D}$-module morphisms $(\mathfrak{D}(r))_{s} \rightarrow(\mathfrak{D}(r))_{\infty}$ map $(\mathfrak{D}(r))_{s}$ isomorphically onto $\mathfrak{D}_{r+s}$. If $F=\bigoplus_{i \in I} \tilde{D}\left(r_{i}\right)$ is a free object of $\mathfrak{R}$, it is natural to let ord $F=$ $\max _{i \in I}\left(-r_{i}\right)$ (called the order of $F$ ) since $\mathfrak{D}(r)$ is free on a single generator of degree $-r$. Clearly $F_{\infty}=\bigoplus_{i \in I} \mathfrak{D}$ since passage to the direct limit commutes with the taking of direct sums. Observe that from the exact sequence

$$
\tilde{\mathfrak{D}}(-k) \otimes F^{*} \rightarrow \tilde{\mathfrak{D}} \otimes E^{*} \rightarrow \tilde{M} \rightarrow 0
$$

we have upon passing to the limit an exact sequence

$$
\mathfrak{D} \otimes F^{*} \rightarrow \mathfrak{D} \otimes E^{*} \rightarrow(\tilde{M})_{\infty} \rightarrow 0 .
$$

Hence the following has been shown.

LEMMA 1. The adjoint cokernel $M$ of the linear differential operator $D$ is $(\tilde{M})_{\infty}$ where $\tilde{M}$ is defined as the cokernel of

$$
\mathfrak{D}(-k) \otimes F^{*} \rightarrow \mathfrak{D} \otimes E^{*} .
$$

In general, call an $\mathfrak{D}$-module $L$ graded if it is written as a direct sum of $\mathfrak{D}$-modules $L=\bigoplus_{p \in Z} L_{p}$. Of course sections of $L_{p}$ will be called homogeneous of degree $p$. This rather artificial motion is useful because of the following lemma. In the statement of the lemma $\otimes L$ is considered as an object of $\Re$ by giving it the obvious left $\tilde{\mathfrak{D}}$-module structure and by letting $(\tilde{D} \otimes L)_{h}=\bigoplus_{p+q=h}(\tilde{D})_{p} \otimes L_{q}$.

LEMMA 2. Let $L$ be a graded $\mathfrak{D}$-module and suppose that every $L_{p}$ is a locally free $\mathfrak{D}$-module. Then $\mathbb{D}$ is a projective object of $\mathfrak{\Re}$.

It will be shown that $P \mapsto \operatorname{Hom}_{\Re}(\tilde{D} \otimes L, P)$ is an exact functor. If $U$ is an open subset of $X$, let Hom $_{\mathcal{O}}(L, P)_{0}(U)$ be $\left\{f \in \operatorname{Hom}_{\mathcal{O}_{\mid U}}(L|U, P| U): f\right.$ maps $L_{d} \mid U$ into $P_{d} \mid U$ for every $\left.d \in Z\right\}$. Then $\operatorname{Hom}_{\mathfrak{O}}(L, P)_{0}$ is a fine sheaf by p. 158 of [3] last 
sentence before 3.8. Now $H^{1}(X$, fine sheaf $)=0[4,2.11 .1$, p. 37]. From an exact sequence $E: 0 \rightarrow P^{\prime} \rightarrow P \rightarrow P^{\prime \prime} \rightarrow 0$ in $\Re$ we obtain an exact sequence $\mathbf{H o m}_{\mathfrak{O}}(L, E)_{0}$ and hence an exact sequence $\Gamma\left(X, \operatorname{Hom}_{\mathfrak{O}}(L, E)_{0}\right)$ since $H^{1}\left(X, \operatorname{Hom}_{\mathfrak{O}}\left(L, P^{\prime}\right)_{0}\right)=0$. Now $\Gamma\left(X, \operatorname{Hom}_{\mathcal{O}}(L, E)_{0}\right)$ can be identified with $\operatorname{Hom}_{\mathfrak{R}}(\tilde{D} \otimes L, E)$ so this last is exact. This proves Lemma 2.

If $L$ is a graded $\mathfrak{D}$-module such that each $L_{p}$ is locally free, the order of $\tilde{D} \otimes L$ will be defined as $\max \left\{r \in Z: L_{r} \neq 0\right\}$ and written ord $(\tilde{D} \otimes L)$. Such an $L$ will be called a locally free graded $\mathfrak{D}$-module.

3. Derivation of the spectral sequences. The manner in which the spectral sequence of [1] was derived motivates the technical considerations that follow. Given an object $P$ of $K$ it will be shown how under certain conditions $\operatorname{Ext}_{\mathfrak{D}}^{p}\left(P_{\infty}, \mathfrak{D}\right)$ can be given as the $p$ th derived functor of certain composite functors. The first of the functors in the composite will now be defined.

Let $\AA^{0}$ be the dual category of $\Re$. A functor $K: \AA^{0} \rightarrow \Re$ will be defined such that if $P$ is any object of $\Re,(K(P))_{-r}=\operatorname{Hom}_{\mathfrak{O}}\left(P_{r}, \mathfrak{D}\right)$ for all $r \in Z$. Defining such an object is equivalent to showing the existence of additive morphisms

$$
\mathfrak{D}_{s} \otimes \operatorname{Hom}_{\mathfrak{O}}\left(P_{r}, \mathfrak{D}\right) \rightarrow \operatorname{Hom}_{\mathfrak{D}}\left(P_{r-s}, \mathfrak{D}\right)
$$

for all $r, s \in Z$ such that all the diagrams

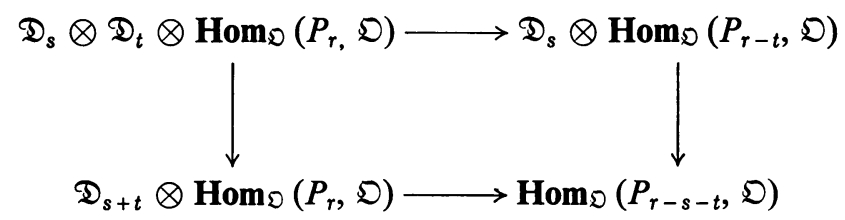

commute. This will be done by first defining an $\mathfrak{D}$-linear morphism

$$
T \otimes \operatorname{Hom}_{\mathfrak{D}}\left(P_{r}, \mathfrak{D}\right) \rightarrow \operatorname{Hom}_{\mathfrak{D}}\left(P_{r-1}, \mathfrak{D}\right) .
$$

Namely if $\gamma \delta$ is a section $\left(^{4}\right)$ of $T$ and $f$ a section of $\mathbf{H o m}_{\mathfrak{O}}\left(P_{r}, \mathfrak{D}\right)$, map $\gamma \delta \otimes f$ to $(\delta \circ f-f \circ \delta) \mid P_{r-1}$, an expression which has meaning because $\delta$ induces an additive endomorphism of $\mathfrak{O}$ (wherever $\delta$ is defined) and also an additive morphism $P_{r-1} \rightarrow P_{r}$ since $\gamma T \subset(\mathfrak{D})_{1}$.

Also if $h$ is any section of $\mathfrak{D}, \gamma h$ maps $P_{r-1}$ into $P_{r}$. Therefore since $\mathfrak{D}_{1}=\mathfrak{D} \oplus T$, we have natural additive morphisms

$$
\mathfrak{D}_{1} \otimes \operatorname{Hom}_{\mathfrak{D}}\left(P_{r}, \mathfrak{D}\right) \rightarrow \operatorname{Hom}_{\mathfrak{D}}\left(P_{r-1}, \mathfrak{D}\right)
$$

(4) It will be left to the reader to fill in the few trivial details that will make arguments of this type entirely rigorous. For instance an open set $U$ over which $\delta$ is defined needs to be given. 
The proof will be complete once it can be shown that we have for all $s \geqq 1$ commutative diagrams of $\mathfrak{D}$-modules

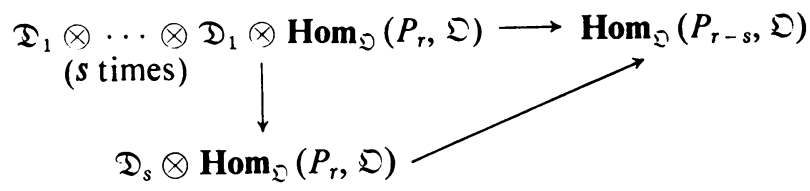

Of course the diagonal arrows are the sought for morphisms and the vertical arrows are given by multiplication. The horizontal arrows are defined inductively from the morphism $(*)$.

Let $L$ be the kernel of

$$
\begin{gathered}
\text { ( } s \text { times }) \\
\mathfrak{D}_{1} \otimes \cdots \otimes \mathfrak{D}_{1} \rightarrow \mathfrak{D}_{s} .
\end{gathered}
$$

It has to be shown that $L \otimes \operatorname{Hom}_{\mathfrak{O}}\left(P_{r}, \mathfrak{D}\right)$ has zero image in $\mathbf{H o m}_{\mathfrak{O}}\left(\boldsymbol{P}_{r-s}, \mathfrak{D}\right)$. The question is local so we may assume that $X$ is an open subset of $\boldsymbol{R}^{n}$ and that $x_{1}, \ldots, x_{n}$ are coordinates on $X$. Modulo elements of the form

$$
\begin{aligned}
& A \otimes \delta \otimes \delta^{\prime} \otimes B-A \otimes \delta^{\prime} \otimes \delta \otimes B-A \otimes 1 \otimes\left[\delta, \delta^{\prime}\right] \otimes B, \\
& A \otimes \delta \otimes a \otimes B-A \otimes a \otimes \delta \otimes B-A \otimes 1 \otimes \delta(a) \otimes B
\end{aligned}
$$

where $A$ is a section of $\mathfrak{D}_{1} \otimes \cdots \otimes \mathfrak{D}_{1}$ ( $t-1$ times), $B$ a section of $\mathfrak{D}_{1} \otimes \cdots \otimes \mathfrak{D}_{1}$ ( $s-t-1$ times), $\delta, \delta^{\prime}$ sections of $T, a$ a section of $\mathcal{D}$, every section of $\mathfrak{D}_{1} \otimes \cdots \otimes \mathscr{D}_{1}$ ( $s$ times) can be written as a linear combination with coefficients in $\mathfrak{D}$ of elements

$$
\begin{aligned}
& 1 \otimes \cdots \otimes 1 \otimes \partial / \partial x_{i(1)} \otimes \cdots \otimes \partial / \partial x_{i(p)} \\
& (s-p \text { times })
\end{aligned}
$$

with $i(1) \leqq i(2) \leqq \cdots \leqq i(p)$. Now the sections $(\dagger)$ are sections of $L$. If $\mathfrak{D}$ is considered as operating on $\mathfrak{D}_{1} \otimes \cdots \otimes \mathfrak{D}_{1}$ ( $s$ times) by left multiplication, the foregoing implies that the elements $(\dagger)$ generate $L$. It is however also clear that if $H$ is any of the sections $(\dagger), H \otimes \operatorname{Hom}_{\mathfrak{O}}\left(M_{r}, \mathfrak{D}\right)$ has zero image in $\operatorname{Hom}_{\mathfrak{O}}\left(M_{r-s}, \mathfrak{D}\right)$.

This completes the proof that the desired morphisms $\mathfrak{D}_{s} \otimes \operatorname{Hom}_{\mathfrak{D}}\left(M_{r}, \mathfrak{D}\right) \rightarrow$ $\operatorname{Hom}_{\mathfrak{O}}\left(M_{r-s}, \mathfrak{D}\right)$ do in fact exist. The fact that $K$ is actually a functor $K: \mathfrak{I}^{0} \rightarrow \mathfrak{\Omega}$ is now clear. Clearly $K$ is left exact.

Let $P$ be an object of $\Re$, and define $C(P)$ to be $\{f \in P(X):$ if $\delta \in T(X), \gamma \delta(f)=0\}$. The elements of $C(P)$ will be called constants of $P$. If $h \in Z$, let $C_{h}(P)=C(P) \cap P_{h}(X)$. The way in which this notion helps us arrive at the desired spectral sequences is suggested by the following lemma.

Lemma 1. Suppose that the object $P$ of $\AA$ has a presentation $F_{p} \rightarrow F_{p-1} \rightarrow \cdots \rightarrow$ $F_{0} \rightarrow P \rightarrow 0$ of length $p$ such that each $F_{p}$ is of the form $\mathfrak{D} \otimes L$ with $L$ a locally free graded $\mathfrak{D}$-module. Let $r=\max _{1 \leqq q \leqq p}\left(\right.$ ord $\left.F_{q}\right)$ and suppose that $r$ is finite. Then 
if $s \geqq r$, $\operatorname{Ext}_{D}^{q}\left(P_{\infty}, \mathfrak{D}\right)$ is the qth derived functor $\left({ }^{5}\right) R^{q}\left(C_{-s} \circ K\right)(P)$ of $C_{-s} \circ K$ for $0 \leqq q \leqq p$.

For $0 \leqq q \leqq p, R^{q}\left(C_{-s} \circ K\right)(P)=H^{q}\left(C_{-s}(K(F)).\right)$ since the $F_{q}$ are injective as objects of $\AA^{0}$. Here of course we use the complex $0 \rightarrow F_{p+1} \rightarrow \cdots \rightarrow F_{0} \rightarrow 0$ with $F_{p+1}$ equal to the kernel of the map $F_{p} \rightarrow F_{p-1}$. Let $f$ be a given element of $C_{-s}\left(K\left(F_{q}\right)\right)$. Write $F_{q}=\tilde{\mathfrak{D}} \otimes L(q \neq p+1)$ with $L$ a locally free graded $\mathfrak{D}$-module. Then $L_{t}=0$ if $t>s$. Now $\left(F_{q}\right)_{\infty}=\mathfrak{D} \otimes L$ and there is a unique $g$ in HomD $\left(\left(F_{q}\right)_{\infty}, \mathfrak{D}\right)$ which agrees with $f$ on $L$. Also $g$ must coincide with $f$ on all of $\left(F_{q}\right)_{s}$. Indeed every section of $\left(F_{q}\right)_{s}$ is (locally) a linear combination with coefficients in $\mathfrak{D}$ of sections of the form $\gamma \delta_{1} \cdots \gamma \delta_{l} \otimes h$ with $\delta_{1}, \ldots, \delta_{l}$ sections of $T$ and $h$ a section of $L_{s-l}$. Since $f$ is a constant,

$$
f\left(\gamma \delta_{1} \cdots \gamma \delta_{l} \otimes h\right)=\gamma \delta_{1}\left(f\left(\gamma \delta_{2} \cdots \gamma \delta_{l} \otimes h\right)\right) .
$$

A similar formula holds with $g$ replacing $f$. Thus the assertion that $f$ and $g$ coincide on $\left(F_{q}\right)_{s}$ follows by induction on $l$.

It follows that every element of $C_{-s}\left(K\left(F_{q}\right)\right)$ has a unique extension to an element of HomD $\left(\left(F_{q}\right)_{\infty}, \mathfrak{D}\right)$. Now $\left(F_{q}\right)_{\infty}$ is a projective of $\mathbb{C}$ for $0 \leqq q \leqq p$ and the sequence $0 \rightarrow\left(F_{p+1}\right)_{\infty} \rightarrow \cdots \rightarrow\left(F_{0}\right)_{\infty} \rightarrow P_{\infty} \rightarrow 0$ is exact. Hence

$$
H^{q}\left(\operatorname{Hom}_{\mathfrak{D}}\left((F .)_{\infty}, \mathfrak{D}\right)\right)=\operatorname{Ext}_{\mathfrak{D}}^{q}\left(P_{\infty}, \mathfrak{D}\right)=H^{q}\left(C_{-s}(K(F .))\right)=R^{q}\left(C_{-s} \circ K\right)(P)
$$

if $0 \leqq q \leqq p$. This proves the lemma.

The following result is the central one.

THEOREM. Let $P$ be any object of $\Re, h \in Z$. There is a spectral sequence which converges to $R^{p+q}\left(C_{-h} \circ K\right)(P)$ and with initial term $E_{2}^{p q}=\left(R^{p} C_{-h} \circ R^{q} K\right)(P)$.

To prove this the theorem on p. 148 of [5] on the spectral sequence for a composite functor will be used. To use that theorem we only need to show that if $P$ is any projective object of $\mathfrak{R},\left(R^{p} C_{-h}\right)(K(P))=0$ if $p>0$. This will be done in the sequence of lemmas that follow. Those lemmas will also help us to determine explicitly the $E_{2}^{p q}$.

Let $\tilde{D}$ be $\oplus_{r \in N} \mathfrak{D}$. It can be considered as an object of $\mathscr{\Re}$ since the $\mathfrak{D}_{r}$ operate on $D$.

LEMMA 2. If $P$ is any object of $\Re,\left(R^{p} C_{s}\right)(P)=\operatorname{Ext}_{\mathscr{\Re}}^{p}(\tilde{\mathfrak{D}}, P(s))$.

First it will be shown that $C_{s}(P)=\operatorname{Hom}_{\Re}(\tilde{\mathfrak{D}}, P(s))$. For this observe that an element $f$ of Hom $\tilde{\mathfrak{D}}(\tilde{\mathfrak{D}}, P(s))$ which is homogeneous of degree zero can be identified with an element $h(f)$ of $P_{s}(X)$ and conversely by matching it with the image under $f$ of 1 in $(\mathfrak{D})_{0}(X)=\mathfrak{D}(X)$. Then $f$ is in $\operatorname{Hom}_{\mathfrak{A}}(\mathfrak{D}, P(s))$ if and only if $h(f)$ is in $C(P)$. To see this, observe that $\tilde{D}$ is a subring of $\mathfrak{D}$ since $\mathfrak{D} \subset \mathfrak{D}_{r}$ for every $r \geqq 0$. Further-

$\left(^{5}\right)$ For the definition of $R^{q}$ see p. 143 of [5]. 
more $\gamma T \subset(\mathfrak{D})_{1}$ generates $\mathfrak{D}$ as an overring of $\tilde{D}$. If $f$ is in $\operatorname{Hom}_{\mathfrak{A}}(\mathfrak{D}, P(s))$, $\gamma \delta(h(f))=\gamma \delta(f(1))=f(\gamma \delta(1))=f(0)=0$ for every $\delta \in T(X)$, so $h(f)$ is a constant. Suppose conversely that $h(f)$ is a constant. If $a \in\left(\tilde{\mathfrak{D}}_{d}\right)(U)$ with $U$ an open subset of $X, f(a)=a(h(f) \mid U)$. If $\delta \in T(U), \quad \gamma \delta(f(a))=\gamma \delta(a(h(f) \mid U))=\gamma \delta(a)(h(f) \mid U)=$ $f(\gamma \delta(a))$ which shows that $f$ is $\mathfrak{D}$-linear.

If $0 \rightarrow P \rightarrow Q^{0} \rightarrow Q^{1} \rightarrow \cdots$ is an injective resolution of $P$ in $\Re, Q(s)$ is then an injective resolution of $P(s)$. Also $\operatorname{Hom}_{\mathscr{R}}(\mathfrak{D}(-s), P)=C_{s}(P)$. It follows that $H^{p}\left(\operatorname{Hom}_{\Re}(\tilde{D}(-s), Q)\right)=\left(R^{p} C_{s}\right)(P)=H^{p}\left(\operatorname{Hom}_{\Re}(\tilde{D}, Q(s))\right)=\operatorname{Ext}_{\mathscr{I}}^{p}(\tilde{\mathfrak{D}}, P(s))$. This completes the proof of the lemma.

The next step is to compute $\left(R^{p} C_{-h}\right)(P)$ for any object $P$ of $\Re$ by using a suitable projective resolution of $\tilde{D}$. Consider $\Lambda^{p} T$ (the $p$ th exterior power of $T$ ) as a graded $\mathfrak{D}$-module by requiring that its sections shall be homogeneous of degree $p$. The object $\tilde{D} \otimes \Lambda^{p} T$ of $\mathfrak{\Re}$ is projective by Lemma 2 of $\S 2$.

LEMmA 3. There exist $\mathfrak{\Re}$-morphisms $\mathfrak{D} \otimes \Lambda^{p} T \rightarrow \mathfrak{D} \otimes \Lambda^{p-1} T$ and a $\mathfrak{A}$-morphism $\mathfrak{D} \rightarrow \tilde{\mathfrak{D}}$ which give a resolution $\tilde{\mathfrak{D}} \otimes \Lambda T \rightarrow \tilde{D} \rightarrow 0$ of $\mathfrak{D}$.

To define $\tilde{D} \otimes \Lambda^{p} T \rightarrow \mathfrak{D} \otimes \Lambda^{p-1} T$ we need to define additive morphisms $\mathfrak{D}_{r} \otimes \Lambda^{p} T \rightarrow \mathfrak{D}_{r+1} \otimes \Lambda^{p-1} T$ for $r \geqq 0$ such that the diagrams

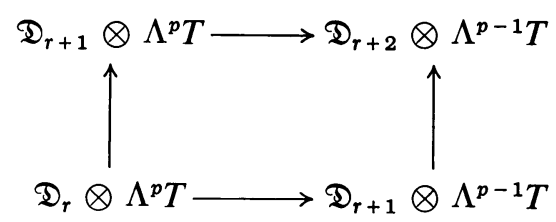

and

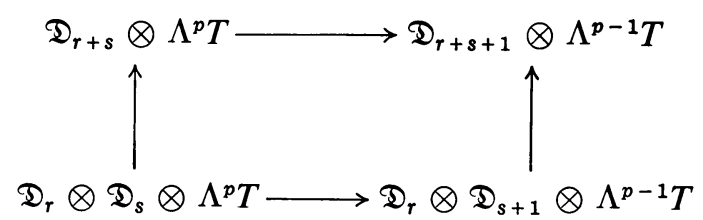

commute. How this can be done has been indicated by Malgrange in [6, p. 23].

LEMMA 4. If $P$ is a projective object of $\Re,\left(R^{q} C_{-h}\right)(K(P))=0$ if $q>0$.

If $P$ is projective, then for an appropriate indexed set of integers $\left(r_{i}\right)_{i \in I}, P$ is a direct summand of $\bigoplus_{i \in I} \tilde{D}\left(r_{i}\right)$. Thus we may assume that $P$ actually is of that form. Then $(K(P))_{-h}=\prod_{i \in I}$ Hom $_{\mathcal{O}}\left(\mathfrak{D}_{h+r_{i}}, \mathfrak{D}\right)$. Hence

$$
\begin{aligned}
& \left(R^{p} C_{-h}\right)(K(P))=\operatorname{Ext}_{\Re}^{p}(\tilde{D}, K(P)(-h))=H^{p}\left(\operatorname{Hom}_{\mathfrak{I}}(\mathfrak{D} \otimes \Lambda \cdot T, K(P)(-h))\right) \\
& =H^{p}\left(\operatorname{Hom}_{\mathcal{O}}\left(\Lambda \cdot T, \prod_{i \in I} \operatorname{Hom}_{\mathcal{O}}\left(\mathfrak{D}_{h+r_{i}-.,} \mathfrak{D}\right)\right)\right) \\
& =H^{p}\left(\Gamma\left(X, \Lambda \cdot T^{*} \otimes \prod_{i \in I} \operatorname{Hom}_{\mathcal{D}}\left(\mathfrak{D}_{h+r_{i}-\cdot,} \mathfrak{D}\right)\right)\right) \text {. }
\end{aligned}
$$


We can consider the factors in $\prod_{i \in I}$ one at a time. In this way we can reduce the problem to showing that all the cohomology groups of the complexes

$0 \rightarrow \Gamma(X, \mathfrak{D}) \rightarrow \Gamma\left(X, \mathbf{H o m}_{\mathfrak{D}}\left(\mathfrak{D}_{h}, \mathfrak{D}\right)\right) \rightarrow \Gamma\left(X, \Lambda^{1} T^{*} \otimes \operatorname{Hom}_{\mathfrak{D}}\left(\mathfrak{D}_{h-1}, \mathfrak{D}\right)\right) \rightarrow \cdots$

are zero.

$$
\rightarrow \Gamma\left(X, \Lambda^{n} T^{*} \otimes \operatorname{Hom}_{\mathcal{O}}\left(\mathcal{D}_{h-n}, \mathfrak{D}\right)\right) \rightarrow 0
$$

Since we are considering sections of fine sheaves we only need to show the sequences

$0 \rightarrow \mathfrak{D} \rightarrow \operatorname{Hom}_{\mathfrak{D}}\left(\mathfrak{D}_{h}, \mathfrak{D}\right) \rightarrow \Lambda^{1} T^{*} \otimes \operatorname{Hom}_{\mathfrak{D}}\left(\mathfrak{D}_{h-1}, \mathfrak{D}\right) \rightarrow \cdots$

$$
\rightarrow \Lambda^{n} T^{*} \otimes \operatorname{Hom}_{\mathcal{D}}\left(\mathfrak{D}_{h-n}, \mathfrak{D}\right) \rightarrow 0
$$

are exact. These are just the sequences (1.3.3) of [2] (with $E=\mathbb{D}$ ) which are known to be exact. This completes the proof of Lemma 4 and of the theorem.

4. The connection with Spencer's theory. Let $E$ and $F$ be vector bundles on the differentiable manifold $X, D: E \rightarrow F$ a linear differential operator. Let $\tilde{M}$ be the object of $\AA$ defined in $\S 2$.

THEOREM. Suppose that $\tilde{M}$ has a presentation

$$
F_{l} \rightarrow F_{l-1} \rightarrow \cdots \rightarrow F_{0} \rightarrow \tilde{M} \rightarrow 0
$$

where each $F_{i}$ is of the form $\mathfrak{D} \otimes L$ with $L$ a locally free graded $\mathfrak{D}$-module of finite type. Let $r=\max _{0 \leqq q \leqq l}$ ord $F_{q}$. Define $R_{h q}=\operatorname{Ext}_{\mathfrak{D}}^{q}\left(\tilde{M}_{h}, \mathfrak{D}\right)$. Then there exist complexes

$$
S_{h q}: 0 \rightarrow R_{h q} \rightarrow \Lambda^{1} T^{*} \otimes R_{h-1, q} \rightarrow \cdots \rightarrow \Lambda^{n} T^{*} \otimes R_{h-n, q} \rightarrow 0
$$

and spectral sequences $\left({ }^{h} E_{r}^{p q}\right)_{r=2,3, \ldots}$ such that

$$
{ }^{n} E_{2}^{p q}=H^{p}\left(\Gamma\left(X, \Lambda \cdot T^{*} \otimes R_{h-\cdot, q}\right)\right) .
$$

If $h \geqq r,\left({ }^{n} E_{r}^{p q}\right)$ converges to $\operatorname{Ext}_{Ð}^{p+q}(M, \mathfrak{D})$ where $M$ is the adjoint cokernel of $D$ for $p+q \leqq l$.

LEMMA. For all $h$ in $Z,\left(R^{q} K\right)(\tilde{M})_{-h}=R_{h q}$.

Let $\cdots \rightarrow P_{1} \rightarrow P_{0} \rightarrow \tilde{M} \rightarrow 0$ be a resolution of $\tilde{M}$ by free objects of $\Omega$. Then $\left(R^{q} K\right)(\tilde{M})$ is $H^{q}(K(P)$.$) and \left(R^{q} K\right)(\tilde{M})_{-h}$ is $H^{q}\left(K(P .)_{-h}\right)=H^{q}\left(\operatorname{Hom}_{\mathfrak{D}}\left((P .)_{h}, \mathfrak{D}\right)\right)$. Now $(P .)_{h}$ is a projective resolution of the $\mathfrak{D}$-module $M_{h}$ since each $\left(P_{q}\right)_{h}$ is locally free and hence projective $\left({ }^{6}\right)$. Hence $\operatorname{Ext}_{\mathfrak{D}}^{q}\left(\tilde{M}_{h}, \mathfrak{D}\right)=H^{q}\left(\operatorname{Hom}_{\mathfrak{D}}\left((P .)_{h}, \mathfrak{D}\right)\right)$. This proves the lemma.

To prove the theorem use first Lemmas 2 and 3 of $\S 3$. They imply that $\left(\left(R^{p} C_{-h}\right) \circ\left(R^{q} K\right)\right)(\tilde{M})$ is $H^{p}\left(\operatorname{Hom}_{\mathcal{A}}\left(\tilde{D} \otimes \Lambda \cdot T,\left(R^{q} K\right)(\tilde{M})(-h)\right)\right)$. Now

$$
\begin{aligned}
\operatorname{Hom}_{\mathfrak{R}}\left(\tilde{\mathfrak{D}} \otimes \Lambda^{p} T,\left(R^{q} K\right)(\tilde{M})(-h)\right) & =\operatorname{Hom}_{\mathfrak{D}}\left(\Lambda^{p} T,\left(R^{q} K\right)(\tilde{M})_{p-h}\right) \\
& =\Gamma\left(X, \Lambda^{p} T^{*} \otimes \mathbf{E x t}_{\mathfrak{D}}^{q}\left(\tilde{M}_{h-p}, \mathfrak{D}\right)\right) \\
& =\Gamma\left(X, \Lambda^{p} T^{*} \otimes R_{h-p, q}\right) .
\end{aligned}
$$

Thus ${ }^{h} E_{2}^{p q}$ has the asserted form.

$\left.{ }^{6}\right)$ One can mimic a part of the proof of Lemma 2 of $\$ 2$ to see this. 
The last statement of the theorem is immediate from Lemma 1 of $\S 3$.

\section{Formally integrable operators.}

THEOREM. Let $E$ and $F$ be vector bundles on a connected differentiable manifold $X, D: E \rightarrow F$ a formally integrable linear differential operator of order $k$. Then the exact sequence

$$
\tilde{\mathfrak{D}}(-k) \otimes F^{*} \rightarrow \mathfrak{D} \otimes E^{*} \rightarrow \tilde{M} \rightarrow 0
$$

can be completed to an infinite resolution

$$
\cdots \rightarrow F_{2} \rightarrow F_{1} \rightarrow F_{0} \rightarrow \tilde{M} \rightarrow 0
$$

in $\mathfrak{N}$ of $\tilde{M}$ where each $F_{i}$ has the form $\mathfrak{D} \otimes L$ with $L$ a graded locally free $\mathfrak{D}$-module of finite type.

The following is the key lemma needed in the proof of this theorem.

Lemma 1. Let $d_{1}, \ldots, d_{p}, e_{1}, \ldots, e_{q} \in Z, A=\mathfrak{D}\left(d_{1}\right) \oplus \cdots \oplus \mathfrak{D}\left(d_{p}\right), B=\mathfrak{D}\left(e_{1}\right) \oplus$ $\cdots \oplus \mathfrak{D}\left(e_{q}\right)$. Let a $\mathfrak{A}$-morphism $\varphi: A \rightarrow B$ be given, and let $P$ be its cokernel. Suppose there exists an integer $k$ such that $P_{k}$ is a vector bundle and for $r>k$, $P_{r} / \gamma P_{r-1}$ is a vector bundle and $\gamma: P_{r-1} \rightarrow P_{r}$ is injective. Then there is an integer $h$ such that the kernel $N$ of $\varphi$ is generated by its sections homogeneous of degree $\leqq h$. The integer $h$ depends only on $d_{1}, \ldots, d_{p}, e_{1}, \ldots, e_{q}$ and $k$.

If $Q$ is any one of the objects $A, B, P$ or $N$ of $\AA$, regard $Q_{\infty}$ as a filtered left $D$-module by letting $Q_{\infty r}$ be the image of $Q_{r}$ in $Q_{\infty}$. The sequence

$$
0 \rightarrow \mathrm{gr}_{r} N_{\infty} \rightarrow \mathrm{gr}_{r} A_{\infty} \rightarrow \mathrm{gr}_{r} B_{\infty} \rightarrow \mathrm{gr}_{r} P_{\infty} \rightarrow 0
$$

is exact for $r>k$ and each term is a vector bundle. The same is true for the sequence

$$
0 \rightarrow N_{\infty k} \rightarrow A_{\infty k} \rightarrow B_{\infty k} \rightarrow P_{\infty k} \rightarrow 0 \text {. }
$$

Given $x \in X$ and an $\mathfrak{D}$-module $G$ on $X$, let $G_{x}$ denote its stalk at $x$. Denote by $G(x)$ the vector space $G_{x} / \mathfrak{m}_{x} G_{x}$ where $\mathfrak{m}_{x}$ is the maximal ideal of $\mathfrak{D}_{x}$. For $r>k$ and $x \in X$ the sequences

$$
0 \rightarrow \operatorname{gr}_{r} N_{\infty}(x) \rightarrow \operatorname{gr}_{r} A_{\infty}(x) \rightarrow \operatorname{gr}_{r} B_{\infty}(x) \rightarrow \operatorname{gr}_{r} P_{\infty}(x) \rightarrow 0
$$

are exact (because $\left.\operatorname{Tor}_{\mathfrak{D}_{x}}^{+}\left(\left(\mathrm{gr}_{r} P_{\infty}\right)_{x}, R\right)=0\right)$. Similarly the sequences

$$
0 \rightarrow N_{\infty k}(x) \rightarrow A_{\infty k}(x) \rightarrow B_{\infty k}(x) \rightarrow P_{\infty k}(x) \rightarrow 0
$$

are exact.

Let $S$ be the graded ring $R\left[X_{1}, \ldots, X_{n}\right]$ where $X_{1}, \ldots, X_{n}$ are indeterminates. The maps gr $A_{\infty}(x) \rightarrow \operatorname{gr} B_{\infty}(x)$ for $x \in X$ are morphisms of the graded $S$-modules

$$
S\left(d_{1}\right) \oplus \cdots \oplus S\left(d_{p}\right) \rightarrow S\left(e_{1}\right) \oplus \cdots \oplus S\left(e_{q}\right) .
$$


It will be shown below that there is an integer $h$ such that the kernels of all such morphisms are generated by their homogeneous parts of degree $\leqq h$. We can and will assume that $h>k$.

Assume that result for the moment and let us see how it follows from this that $N$ is generated by its homogeneous parts of degree $\leqq h$. Let $r \geqq h$ and observe that given $x \in X$ and $\xi$ in $\left(\operatorname{gr}_{r} N_{\infty}\right)_{x}$, there exist $\xi_{1}, \ldots, \xi_{m}$ in $\left(\mathrm{gr}_{h} N_{\infty}\right)_{x}$ and $A_{1}, \ldots, A_{m}$ in $\left(\mathrm{gr}_{r-h}(\mathcal{D})_{x}\right.$ such that $A_{1} \xi_{1}+\cdots+A_{m} \xi_{m}$ and $\xi$ have the same image in $\operatorname{gr}_{r} N_{\infty}(x)$. Now $\left(\mathrm{gr}_{r} N_{\infty}\right)_{x}$ is a module of finite type over $\mathfrak{D}_{x}$. Hence Nakayama's lemma implies that $\xi$ actually has the form $A_{1} \xi_{1}+\cdots+A_{m} \xi_{m}$ (although we might have to take a different choice of the $A_{i}$ and $\xi_{i}$ ).

We therefore have that $\mathrm{gr} N_{\infty}$ is generated by its homogeneous parts of degree $\leqq h$. From this it follows easily enough that $N$ is generated by its homogeneous parts of degree $\leqq h$.

In the course of the preceding proof, the following lemma was used.

Lemma 2. Let $d_{1}, \ldots, d_{p}, e_{1}, \ldots, e_{q} \in Z, X_{1}, \ldots, X_{n}$ be indeterminates and $S=\boldsymbol{R}\left[X_{1}, \ldots, X_{n}\right]$. Let $I$ be the graded $S$-module $I=S\left(d_{1}\right) \oplus \cdots \oplus S\left(d_{p}\right)$ and $H$ the graded $S$-module $H=S\left(e_{1}\right) \oplus \cdots \oplus S\left(e_{q}\right)$. There exists an integer $h$ such that if $\varphi: I \rightarrow H$ is any (degree 0$)$ morphism of graded $S$-modules, the $S$-module $\operatorname{ker} \varphi$ is generated by its homogeneous parts of degree $\leqq h$.

Let us defer the proof of this lemma until the proof of the theorem is completed. Assume that we have obtained a presentation of length $p$ of $\tilde{M}$

$$
F_{p} \rightarrow \cdots \rightarrow F_{1} \rightarrow F_{0} \rightarrow \tilde{M} \rightarrow 0
$$

with $F_{1} \rightarrow F_{0}$ as specified in the theorem. We need to see that it can be extended to a presentation of length $p+1$. Let $A=F_{p}, B=F_{p-1}$ and let $P$ be the cokernel of the given morphism $A \rightarrow B$. Locally the situation is the same as in Lemma 1 and with the same $k$ as in the statement of the theorem. Also since $X$ is connected, the $d$ 's and $e$ 's do not change from place to place. We can therefore conclude that there exists an $h$ such that the kernel $N$ of $F_{p} \rightarrow F_{p-1}$ is generated by its terms of degree $\leqq h$. Now for every integer $m, N_{m}$ is a vector bundle. Let $L=\bigoplus_{m \leqq h} N_{m}$. Let $F_{p+1}=\mathfrak{D} \otimes L$. There is a unique $\Re$-morphism $F_{p+1} \rightarrow F_{p}$ whose restriction to $L$ coincides with the inclusion of $L$ in $F_{p}$. This proves the theorem.

A brief outline of the proof of Lemma 2 based on [7] and [8] will now be given.

Consider the coherent modules $\Im=\left(C \otimes_{R} I\right)^{\sim}$ and $\mathfrak{S}=\left(C \otimes_{R} H\right)^{\sim}$ on the projective scheme $Y=\boldsymbol{P}_{C}^{n-1}$. By Proposition 1.2 of [7] the family $E$ of kernels of morphisms $\mathfrak{\Im} \rightarrow \mathfrak{H}$ is bounded. Theorem 2.1 of [7] says these can all be written as quotients of $O_{Y}(-r)^{N}$ for a fixed pair of integers $r$ and $N$. Consider the family $E^{\prime}$ of kernels of morphisms from $O_{Y}(-r)^{N}$ to a member of $E$. Using induction on $N$, Theorem 2.1 of [7] and the theorem of [8], it follows that there is an integer $u$ such that if $v \geqq u, H^{t}(Y, \mathfrak{F}(v))=0$ whenever $t>0$ and $\mathfrak{F}$ is an element of $E^{\prime}$. 
Let $h=\max (u, r)$. A morphism $\varphi: I \rightarrow H$ induces a morphism $\tilde{\varphi}: \mathfrak{\Im} \rightarrow \mathfrak{F}$. Let $A$ be the kernel of $\varphi$. Then $\tilde{A}$ is the kernel of $\tilde{\varphi}$ and we have a morphism $O_{Y}(-r)^{N} \rightarrow$ $\tilde{A}$. Let $\mathfrak{F}$ be its kernel. Now if $i \geqq h, S(-r)_{i}^{N}=\Gamma\left(Y, O_{Y}(i-r)^{N}\right) \rightarrow \Gamma(Y, \tilde{A}(i))=A_{i}$ is surjective because $H^{1}(Y, \mathfrak{F}(i))=0$. This completes the proof since $S(-r)^{N}$ is generated by its elements homogeneous of degree $\leqq r$.

\section{BIBLIOGRAPHY}

1. Joseph Johnson, Some homological properties of Spencer's cohomology theory, J. Differential Geometry (to appear).

2. Donald C. Spencer, Overdetermined systems of linear partial differential equations, Bull. Amer. Math. Soc. 75 (1969), 179-239. MR 39 \#3533.

3. Roger Godement, Topologie algébrique et théorie des faisceaux, Actualités Sci. Indust., no. 1252, Hermann, Paris, 1958. MR 21 \#1583.

4. F. Hirzebruch, Neue topologische Methoden in der algebraischen Geometrie, Ergebnisse der Math. und Grenzgebiete, Heft 9, Springer-Verlag, Berlin, 1962; English transl., Die Grundlehren der math. Wissenschaften, Band 131, Springer-Verlag, New York, 1966. MR 25 \#1155; MR 34 \#2573.

5. Alexander Grothendieck, Sur quelques points d'algèbre homologique, Tôhoku Math. J. (2) 9 (1957), 119-221. MR 21 \#1328.

6. Bernard Malgrange, Cohomologie de Spencer (d'après Quillen), Publications du séminaire mathématique, d'Orsay, 1966.

7. Alexander Grothendieck, Techniques de construction et théorèmes d'existence en géométrie algébrique. IV: Les schémas de Hilbert, Séminaire Bourbaki 1960/61, Exposés 221, Secrétariat mathématique, Paris, 1961. MR 27 \#1339.

8. David Mumford, Lectures on curves on an algebraic surface, Ann. of Math. Studies, no. 59, Princeton Univ. Press, Princeton, N. J., 1966, Lecture no. 14. MR 35 \#187.

RUTGERS UNIVERSITY,

New Brunswick, New Jersey 08903 